\title{
Cartesian Products of Some Regular Graphs Admitting Antimagic Labeling for Arbitrary Sets of Real Numbers
}

\author{
Yi-Wu Chang $(\mathbb{D})$ and Shan-Pang Liu \\ Department of Mathematical Sciences, National Chengchi University, Taipei, Taiwan \\ Correspondence should be addressed to Yi-Wu Chang; chang@nccu.edu.tw
}

Received 29 April 2021; Revised 17 October 2021; Accepted 26 November 2021; Published 31 December 2021

Academic Editor: M. M. Bhatti

Copyright (c) 2021 Yi-Wu Chang and Shan-Pang Liu. This is an open access article distributed under the Creative Commons Attribution License, which permits unrestricted use, distribution, and reproduction in any medium, provided the original work is properly cited.

An edge labeling of graph $G$ with labels in $A$ is an injection from $E(G)$ to $A$, where $E(G)$ is the edge set of $G$, and $A$ is a subset of $\mathbb{R}$. A graph $G$ is called $\mathbb{R}$-antimagic if for each subset $A$ of $\mathbb{R}$ with $|A|=|E(G)|$, there is an edge labeling with labels in $A$ such that the sums of the labels assigned to edges incident to distinct vertices are different. The main result of this paper is that the Cartesian products of complete graphs (except $K_{1}$ ) and cycles are $\mathbb{R}$-antimagic.

\section{Introduction}

All graphs considered in this paper are finite, simple, and without isolated vertices. As usual, let $\mathbb{R}$ denote the set of real numbers. For a graph $G$ and a vertex $v$ in $G, V(G), E(G)$, and $E_{G}(v)$ denote the vertex set of $G$, the edge set of $G$, and the set of edges incident to $v$ in $G$, respectively. In this paper, the following terminologies and notations are used. Let $G$ be a graph. When $A$ is a subset of $\mathbb{R}$ with $|A|=|E(G)|$ and the function $f: E(G) \longrightarrow A$ is injective, we say that $f$ is an edge labeling of $G$ with labels in $A$; in this case, for any vertex $v$ of $G$, we use $f^{+}(v)$ to denote $\sum_{e \in E_{G}(v)} f(e)$. If $B$ is a subset of $\mathbb{R}$ with $|B| \geq|E(G)|$ such that for each subset $A$ of $B$ with $|A|=|E(G)|$, there is an edge labeling of $G$ with labels in $A$ such that $f^{+}(u)$ is not equal to $f^{+}(v)$ for any two distinct vertices $u, v$ of $G$, then we say that $G$ is $B$-antimagic.

In the literature, a graph $G$ is antimagic if $G$ is $\{1,2, \ldots,|E(G)|\}$-antimagic. The concept of antimagic graphs was introduced by Hartsfield and Ringel [1] in 1990. They conjectured that every connected graph with at least two edges was antimagic. This conjecture has not been completely solved yet. Some partial results are listed below. The antimagicness for some special types of regular graphs is verified by Cranston [2], Cranston et al. [3], and Liang and
Zhu [4]. Then, Chang et al. [5] proved that all regular graphs with degree $\geq 2$ are antimagic.

Some studies have addressed the antimagicness of Cartesian products. In 2008, Wang and Hsiao [6] introduced new classes of antimagic graphs constructed through Cartesian products, and Wang [7] proved that any Cartesian product of two or more cycles is antimagic. The antimagicness of the Cartesian products of two paths and the Cartesian products of two or more regular graphs are proved in $[8,9]$ by Cheng. Moreover, Zhang and Sun [10] proved that if a regular graph $G$ is antimagic, then for any connected graph $H$, the Cartesian product $G \square H$ is antimagic.

Let $\mathbb{R}^{+}$denote the set of real numbers. A graph $G$ is universal antimagic if $G$ is $\mathbb{R}^{+}$-antimagic. Matamala and Zamora [11] proved that paths, cycles, and graphs whose connected components are cycles or paths of odd lengths are universal antimagic in 2020. In this paper, we generalize further and define $\mathbb{R}$-antimagic graphs. The methods of labeling on Cartesian products of cycles used in this paper are similar in $[7,8]$. In Section 2, we show that wheels, cycles, and complete graphs of order $\geq 3$ are $\mathbb{R}$-antimagic. In Section 3, we show that Cartesian products $G_{1} \square G_{2} \square \cdots \square G_{n}(n \geq 2)$ are $\mathbb{R}$-antimagic, where each $G_{i}$ is a complete graph of order $\geq 2$ or a cycle. 


\section{2. $\mathbb{R}$-Antimagic Graphs and Uniformly $\mathbb{R}$-Antimagic Graphs}

Let $P_{n}$ be a path on $n$ vertices. In [11], it is shown that $P_{n}(n \geq 3)$ is $\mathbb{R}^{+}$-antimagic, but $P_{3}, P_{4}, P_{5}$ are not $\mathbb{R}$-antimagic. Shang et al. [12] investigated the antimagicness of star forests. We prove that stars are $\mathbb{R}^{+}$-antimagic, but not $\mathbb{R}$-antimagic.

Remark 1. Let $S_{n}$ denote the star with $n$ edges. Then, $S_{n}(n \geq 2)$ is $\mathbb{R}^{+}$-antimagic, but not $\mathbb{R}$-antimagic.

Proof. Let $S_{n}$ be the star with $V\left(S_{n}\right)=\left\{v_{1}, v_{2}, \ldots, v_{n}\right\} \cup\{v\}$ and $E\left(S_{n}\right)=\left\{v v_{i} \mid i=1,2, \ldots, n\right\}$.

Let $r_{1}<r_{2}<r_{3}<\cdots<r_{n}$ be the arbitrarily given positive numbers. We define an edge labeling $f$ of $S_{n}$ with labels in $\left\{r_{1}, r_{2}, r_{3}, \ldots, r_{n}\right\}$ by $f\left(v v_{i}\right)=r_{i}$ for $i=1,2, \ldots, n$. Then,

$$
f^{+}\left(v_{i}\right)=r_{i}<r_{i+1}=f^{+}\left(v_{i+1}\right) \text {, }
$$

for $i=1,2, \ldots, n-1$, and

$$
f^{+}\left(v_{n}\right)=r_{n}<r_{1}+r_{2}+\cdots+r_{n}=f^{+}(v) .
$$

We have

$$
f^{+}\left(v_{1}\right)<f^{+}\left(v_{2}\right)<\cdots<f^{+}\left(v_{n}\right)<f^{+}(v) .
$$

Hence, $S_{n}$ is $\mathbb{R}^{+}$-antimagic.

Let $r_{1}<r_{2}<r_{3}<\cdots<r_{n}$ be real numbers with $r_{1}+r_{2}+\cdots+r_{n-1}=0$. Let $f$ be an arbitrary edge labeling of $S_{n}$ with labels in $\left\{r_{1}, r_{2}, r_{3}, \ldots, r_{n}\right\}$. Without loss of generality, $f$ is defined by $f\left(v v_{i}\right)=r_{i}$ for $i=1,2, \ldots, n$. We see that $f^{+}\left(v_{n}\right)=r_{n}=r_{1}+r_{2}+\cdots+r_{n-1}+r_{n}=f^{+}(v)$. Accordingly, $S_{n}$ is not $\left\{r_{1}, r_{2}, r_{3}, \ldots, r_{n}\right\}$-antimagic, which results in $S_{n}$ not $\mathbb{R}$-antimagic.

Let $K_{n}$ denote the complete graph of $\operatorname{order} n$, and $C_{n}$ the cycle of order $n$. A wheel $W_{n}(n \geq 3)$ is the graph obtained by connecting a single vertex to every vertex of the cycle $C_{n}$. In this section, we prove that wheels, cycles, and complete graphs of order $\geq 3$ are $\mathbb{R}$-antimagic.

Theorem 1. Every wheel is $\mathbb{R}$-antimagic.

Proof. Let $W_{n}$ be the wheel with $V\left(W_{n}\right)=\left\{v_{1}, v_{2}, \ldots, v_{n}\right\}$ $\cup\{v\}$ and $E\left(W_{n}\right)=\left\{v_{1} v_{2}\right\} \cup\left\{v_{i} v_{i+2} \mid i=1,2, \ldots, n-2\right\} \cup\left\{v_{n}\right.$ $\left.-1 v_{n}\right\} \cup\left\{v v_{i} \mid i=1,2, \ldots, n\right\}$. To prove the theorem, let $r_{1}<r_{2}<r_{3}<\cdots<r_{2 n}$ be the arbitrarily given real numbers. We distinguish two cases: Case $1, r_{n-1}+r_{n}<r_{n+1}+r_{n+2}+\cdots$ $+r_{2 n-1}$, and Case 2, $r_{n+1}+r_{n+2}+\cdots+r_{2 n-1} \leq r_{n-1}+r_{n}$.

Case 1. $r_{n-1}+r_{n}<r_{n+1}+r_{n+2}+\cdots+r_{2 n-1}$.

We define an edge labeling $f$ of $W_{n}$ with labels in $\left\{r_{1}, r_{2}, r_{3}, \ldots, r_{2 n}\right\}$ by $f\left(v_{1} v_{2}\right)=r_{1}, f\left(v_{i} v_{i+2}\right)=r_{i+1}$ for $i=1,2, \ldots, n-2, f\left(v_{n-1} v_{n}\right)=r_{n}$, and $f\left(v v_{i}\right)=r_{n+i}$ for $i=1,2$, $\ldots, n$ (see Figure 1). Then, $f^{+}\left(v_{1}\right)=r_{1}+r_{2}+r_{n+1}, f^{+}\left(v_{i}\right)=$ $r_{i-1}+r_{i+1}+r_{n+i}$ for $i=2, \ldots, n-1$, and $f^{+}\left(v_{n}\right)=r_{n-1}+r_{n}+r_{2 n}$. Note that

$$
\begin{aligned}
& f^{+}\left(v_{1}\right)=r_{1}+r_{2}+r_{n+1}<r_{1}+r_{3}+r_{n+2}=f^{+}\left(v_{2}\right) \\
& f^{+}\left(v_{i}\right)=r_{i-1}+r_{i+1}+r_{n+i}<r_{i}+r_{i+2}+r_{n+i+1}=f^{+}\left(v_{i+1}\right)
\end{aligned}
$$

for $i=2, \ldots, n-2$,

$$
\begin{aligned}
f^{+}\left(v_{n-1}\right)= & r_{n-2}+r_{n}+r_{2 n-1}<r_{n-1}+r_{n}+r_{2 n}=f^{+}\left(v_{n}\right), \\
f^{+}\left(v_{n}\right)= & \left(r_{n-1}+r_{n}\right)+r_{2 n}<\left(r_{n+1}+r_{n+2}+\cdots+r_{2 n-1}\right) \\
& +r_{2 n}=f^{+}(v) .
\end{aligned}
$$

Hence,

$$
f^{+}\left(v_{1}\right)<f^{+}\left(v_{2}\right)<\cdots<f^{+}\left(v_{n}\right)<f^{+}(v) .
$$

Case 2. $r_{n+1}+r_{n+2}+\cdots+r_{2 n-1} \leq r_{n-1}+r_{n}$.

We define an edge labeling $f$ of $W_{n}$ with labels in $\left\{r_{1}, r_{2}, r_{3}, \ldots, r_{2 n}\right\}$ by $f\left(v_{1} v_{2}\right)=r_{n+1}, f\left(v_{i} v_{i+2}\right)=r_{n+i+1}$ for $i=1,2, \ldots, n-2, f\left(v_{n-1} v_{n}\right)=r_{2 n}$, and $f\left(v v_{i}\right)=r_{i}$ for $i=1$, $2, \ldots, n$ (see Figure 2). Then, $f^{+}\left(v_{1}\right)=r_{n+1}+r_{n+2}+r_{1}$, $f^{+}\left(v_{i}\right)=r_{n+i-1}+r_{n+i+1}+r_{i}$ for $i=2, \ldots, n-1$, and $f^{+}\left(v_{n}\right)=r_{2 n-1}+r_{2 n}+r_{n}$. Note that

$$
\begin{aligned}
& f^{+}\left(v_{1}\right)=r_{n+1}+r_{n+2}+r_{1}<r_{n+1}+r_{n+3}+r_{2}=f^{+}\left(v_{2}\right), \\
& f^{+}\left(v_{i}\right)=r_{n+i-1}+r_{n+i+1}+r_{i}<r_{n+i}+r_{n+i+2}+r_{i+1}=f^{+}\left(v_{i+1}\right),
\end{aligned}
$$

for $i=2, \ldots, n-2$,

$$
\begin{aligned}
f^{+}\left(v_{n-1}\right) & =r_{2 n-2}+r_{2 n}+r_{n-1}<r_{2 n-1}+r_{2 n}+r_{n}=f^{+}\left(v_{n}\right) \\
f^{+}(v)= & r_{1}+\left(r_{2}+r_{3}+\cdots+r_{n-1}+r_{n}\right) \\
< & r_{1}+\left(r_{n+1}+r_{n+2}+\cdots+r_{2 n-2}+r_{2 n-1}\right) \leq r_{1} \\
& +\left(r_{n-1}+r_{n}\right)=f^{+}\left(v_{1}\right) .
\end{aligned}
$$

Hence,

$$
f^{+}(v)<f^{+}\left(v_{1}\right)<f^{+}\left(v_{2}\right)<\cdots<f^{+}\left(v_{n}\right) .
$$

This completes the proof.

To prove the results in Section 3, we need the concept of uniformly $\mathbb{R}$-antimagic graphs, which is defined below. Let $G$ be a graph. Suppose that all the vertices of $G$ can be listed as $u_{1}, u_{2}, \ldots, u_{m}$ such that for every $A \subseteq \mathbb{R}$ with $|A|=|E(G)|$, there is an edge labeling $f$ of $G$ with labels in $A$ such that $f^{+}\left(u_{1}\right)<f^{+}\left(u_{2}\right)<\cdots<f^{+}\left(u_{m}\right)$. Then, we say that $G$ is uniformly $\mathbb{R}$-antimagic and that the sequence of vertices $u_{1}, u_{2}, \ldots, u_{m}$ has the uniformly $\mathbb{R}$-antimagic property. Note that in this definition, the ordering of the vertices $u_{1}, u_{2}, \ldots, u_{m}$ satisfying the property $f^{+}\left(u_{1}\right)$ $<f^{+}\left(u_{2}\right)<\cdots<f^{+}\left(u_{m}\right)$ is independent of the choice of the subset $A$ of $\mathbb{R}$. Obviously, every uniformly $\mathbb{R}$-antimagic graph is $\mathbb{R}$-antimagic.

Before proving our main result, we first describe uniformly $\mathbb{R}$-antimagic property with cycles and complete graphs. 


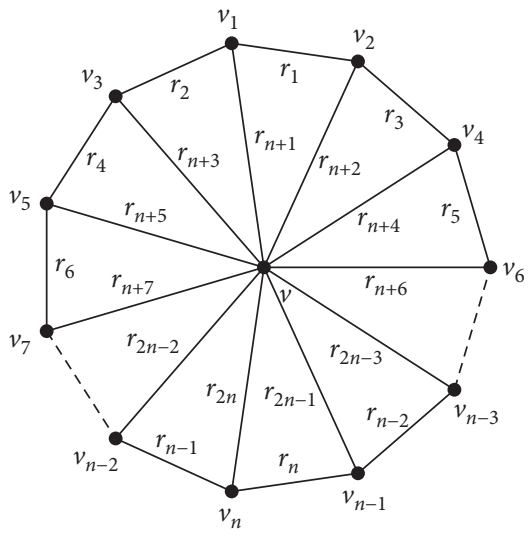

$n$ is odd

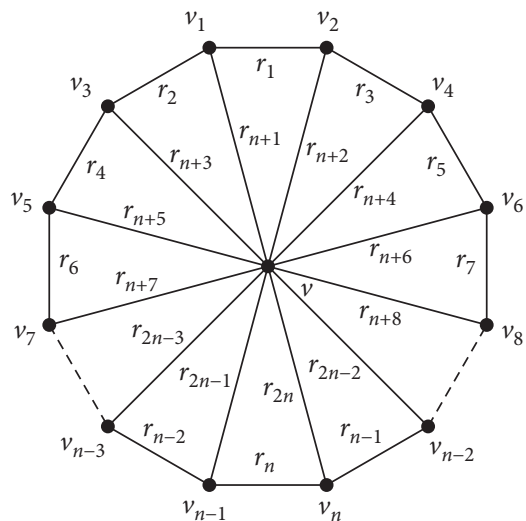

$n$ is even

FIGURE 1: Edge labeling of $W_{n}$ if $r_{n-1}+r_{n}<r_{n+1}+r_{n+2}+\cdots+r_{2 n-1}$.

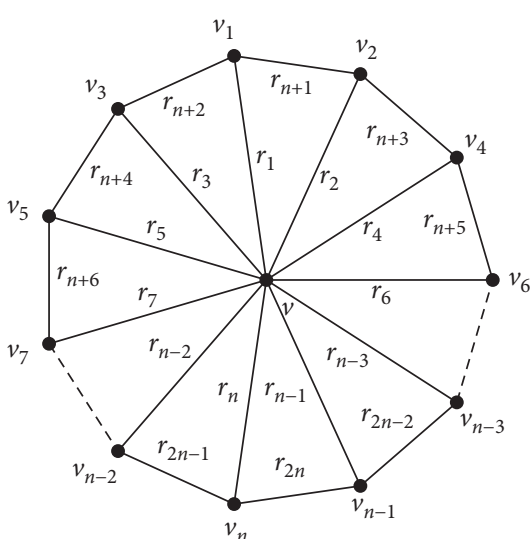

$n$ is odd

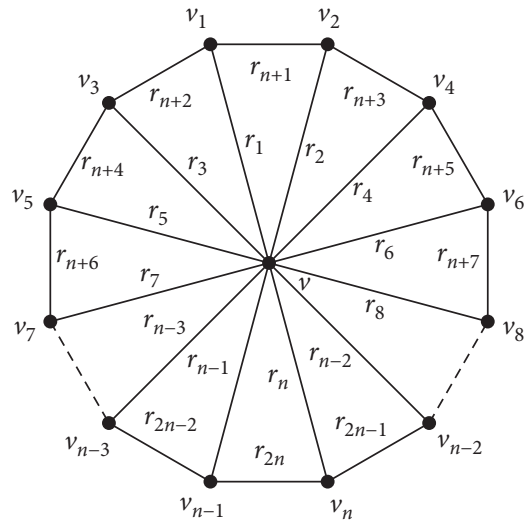

$n$ is even

Figure 2: Edge labeling of $W_{n}$ if $r_{n+1}+r_{n+2}+\cdots+r_{2 n-1} \leq r_{n-1}+r_{n}$.

Theorem 2. Every cycle is uniformly $\mathbb{R}$-antimagic.

Proof. Let $C_{n}$ be the cycle with vertex set $\left\{v_{1}, v_{2}, \ldots, v_{n}\right\}$ and edge set $\left\{v_{1} v_{2}\right\} \cup\left\{v_{i} v_{i+2} \mid i=1,2, \ldots, n-2\right\} \cup\left\{v_{n-1} v_{n}\right\}$. Let $r_{1}<r_{2}<r_{3}<\cdots<r_{n}$ be the arbitrarily given $n$ real numbers. We define an edge labeling $f$ of $C_{n}$ with labels in $\left\{r_{1}, r_{2}, \ldots, r_{n}\right\}$ by $f\left(v_{1} v_{2}\right)=r_{1}, \quad f\left(v_{i} v_{i+2}\right)=r_{i+1}$ for $i=1,2, \ldots, n-2$, and $f\left(v_{n-1} v_{n}\right)=r_{n}$ (see Figure 3).

Then, $f^{+}\left(v_{1}\right)=r_{1}+r_{2}, f^{+}\left(v_{i}\right)=r_{i-1}+r_{i+1}$ for $i=2, \ldots$, $n-1$, and $f^{+}\left(v_{n}\right)=r_{n-1}+r_{n}$. Since $r_{1}+r_{2}<r_{1}+r_{3}<r_{2}$ $+r_{4}<r_{3}+r_{5}<r_{4}+r_{6}<\cdots<r_{n-3}+r_{n-1}<r_{n-2}+r_{n}<r_{n-1}+$ $r_{n}$, we have $f^{+}\left(v_{1}\right)<f^{+}\left(v_{2}\right)<\cdots<f^{+}\left(v_{n}\right)$. We see that the listing of vertices $v_{1}, v_{2}, \ldots, v_{n}$ with the property $f^{+}\left(v_{1}\right)<$ $f^{+}\left(v_{2}\right)<\cdots<f^{+}\left(v_{n}\right)$ is independent of the arbitrarily given $r_{1}<r_{2}<r_{3}<\cdots<r_{n}$. Thus, $C_{n}$ is uniformly $\mathbb{R}$-antimagic.

Theorem 3. The complete graph $K_{n}(n \geq 3)$ is uniformly $\mathbb{R}$-antimagic.

Proof. Let $K_{n}$ be the complete graph with vertex set $V\left(K_{n}\right)$ $=\left\{v_{1}, v_{2}, \ldots, v_{n}\right\}$ and edge set $E\left(K_{n}\right)=\left\{v_{i} v_{j} \mid 1 \leq i \leq j \leq n\right\}$. Let $r_{1}<r_{2}<r_{3}<\cdots<r_{\left(\begin{array}{c}n \\ 2\end{array}\right)}$ be the arbitrarily given real numbers.
Let $f$ be an edge labeling of $K_{n}$ with labels in $\left\{r_{1}, r_{2}, r_{3}, \ldots, r_{\left.\left(\begin{array}{c}n \\ 2\end{array}\right)\right\}}\right\}$ such that for $i=1,2, \ldots, n-2$, $f\left(v_{i} v_{i+1}\right)<f\left(v_{i} v_{i+2}\right)<f\left(v_{i} v_{i+3}\right)<\cdots<f \quad\left(v_{i} v_{n}\right)<f \quad\left(v_{i+1}\right.$ $\left.v_{i+2}\right)$. Hence, $f\left(v_{1} v_{2}\right)<f\left(v_{1} v_{3}\right)<\cdots<f\left(v_{1} v_{n}\right)<f\left(v_{2} v_{3}\right)$ $<f\left(v_{2} v_{4}\right)<\cdots<f\left(v_{2} v_{n}\right)<f\left(v_{3} v_{4}\right)<\cdots<f\left(v_{n-1} v_{n}\right)$.

For $1 \leq i \leq n-1$, we have

$$
\begin{aligned}
f^{+}\left(v_{i}\right)= & \sum_{1 \leq k<i} f\left(v_{k} v_{i}\right)+f\left(v_{i} v_{i+1}\right)+\sum_{i+1<k \leq n} f\left(v_{i} v_{k}\right) \\
< & \sum_{1 \leq k<i} f\left(v_{k} v_{i+1}\right)+f\left(v_{i} v_{i+1}\right) \\
& +\sum_{i+1<k \leq n} f\left(v_{i+1} v_{k}\right)=f^{+}\left(v_{i+1}\right) .
\end{aligned}
$$

Hence, $f^{+}\left(v_{1}\right)<f^{+}\left(v_{2}\right)<\cdots<f^{+}\left(v_{n}\right)$. We see that the listing of vertices $v_{1}, v_{2}, \ldots, v_{n}$ with the property $f^{+}\left(v_{1}\right)<f^{+}\left(v_{2}\right)<\cdots<f^{+}\left(v_{n}\right)$ is independent of the arbitrarily given $r_{1}<r_{2}<r_{3}<\cdots<r_{\left(\begin{array}{c}n \\ 2\end{array}\right)}$. Thus, $K_{n}$ is uniformly $\mathbb{R}$-antimagic. 

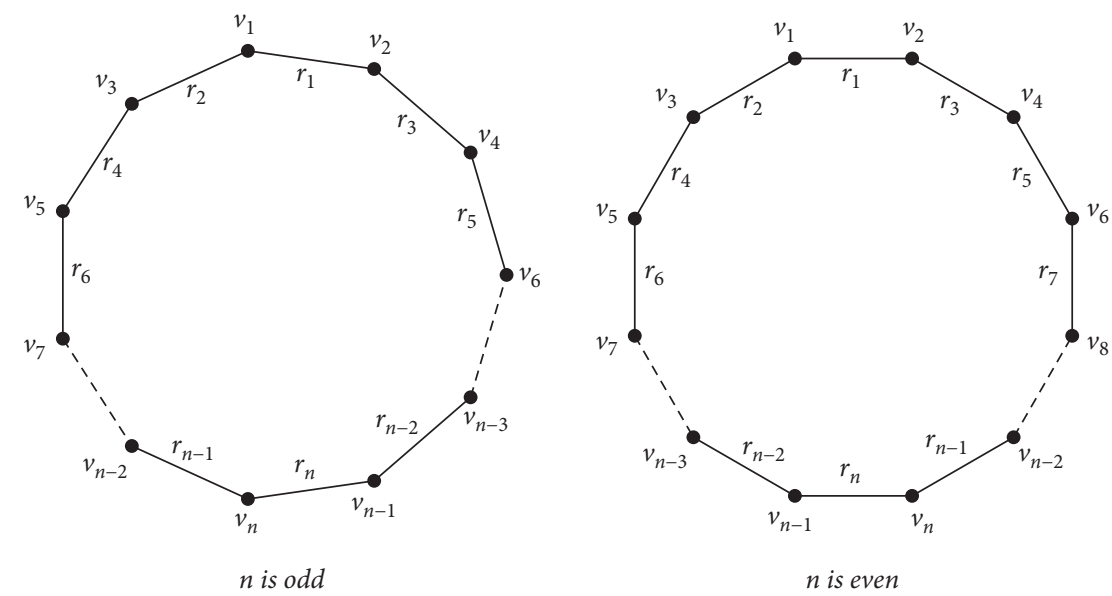

Figure 3: Edge labeling of $C_{n}$.

\section{Main Results}

Let $G$ be a graph and $A$ be a subset of $\mathbb{R}$ with $|A|=|E(G)|$. If $g$ is an edge labeling of $G$ with labels in $A$ and $K, L$ are nonempty subsets of $E(G)$ such that $g(x)<g(y)$ for all $x \in K, y \in L$, then we write $K \prec L$ under $g$. It is easy to see that the relation $\prec$ is transitive (i.e., if $K, L, M$ are nonempty subsets of $E(G)$, and $K \prec L, L \prec M$, then $K \prec M)$. The following trivial lemma will be used in the proofs of Theorems 4 and 5 .

Lemma 1. Let $G$ be an arbitrary graph and $A$ be a subset of $\mathbb{R}$ with $|A|=|E(G)|$. Let $g$ be an edge labeling of $G$ with labels in A. Suppose that $A_{1}, A_{2}, B_{1}, B_{2}$ are pairwise disjoint nonempty subsets of the edge set $E(G)$ with $\left|A_{1}\right|=\left|B_{1}\right|,\left|A_{2}\right|=\left|B_{2}\right|=1$ such that $A_{1} \prec B_{1} \cup B_{2}$ and $A_{2} \prec B_{1}$ under g. Then,

$$
\sum_{e \in A_{1} \cup A_{2}} g(e)<\sum_{e \in B_{1} \cup B_{2}} g(e) .
$$

Proof. Let $A_{2}=\{a\}$ and $b$ be an arbitrary edge in $B_{1}$. Since $A_{2} \prec B_{1}$ under $g$, we have $g(a)<g(b)$. Since $A_{1} \prec B_{1} \cup B_{2}$ under $g$ and $\left|A_{1}\right|=\left|B_{2} \cup\left(B_{1}-\{b\}\right)\right|$, we have

$$
\sum_{e \in A_{1}} g(e)<\sum_{e \in B_{2} \cup\left(B_{1}-\{b\}\right)} g(e) .
$$

Note that

$$
\sum_{e \in A_{1} \cup A_{2}} g(e)=g(a)+\sum_{e \in A_{1}} g(e),
$$

and

$$
\sum_{e \in B_{1} \cup B_{2}} g(e)=g(b)+\sum_{e \in B_{2} \cup\left(B_{1}-\{b\}\right)} g(e) .
$$

Combining (12)-(14) and $g(a)<g(b)$, we have

$$
\sum_{e \in A_{1} \cup A_{2}} g(e)<\sum_{e \in B_{1} \cup B_{2}} g(e) .
$$

We need the following notations. Let $G$ be a graph, and $A$ be a subset of $\mathbb{R}$ with $|A|=|E(G)|$. If $f$ is an edge labeling of $G$ with labels in $A$ and $D$ being a nontrivial connected subgraph of $G$ which contains no isolated vertices, then we use $f_{E(D)}$ to denote the restriction of $f$ to $E(D)$ with range $f(E(D))$. Obviously, $f_{E(D)}$ is an edge labeling of $D$ with labels in $f(E(D))$. Moreover, for a vertex $v \in V(D)$, we use $f_{E(D)}^{+}(v)$ to denote $\left(f_{E(D)}\right)^{+}(v)$. Recall that $E_{D}(v)$ is the set of all edges incident to $v$ in $D$. Thus, $f_{E(D)}^{+}(v)$ $=\sum_{e \in E_{D}(v)} f(e)$.

Let $G$ and $H$ be two graphs with $V(G)=\left\{u_{1}, u_{2}, \ldots, u_{m}\right\}$ and $V(H)=\left\{v_{1}, v_{2}, \ldots, v_{n}\right\}$, respectively. The Cartesian product of $G$ and $H$, denoted by $G \square H$, is the graph with vertex set $V(G) \times V(H)$ such that $\left(u_{i}, v_{j}\right)$ is adjacent to $\left(u_{k}, v_{l}\right)$ if either $u_{i}=u_{k}$ and $v_{j} v_{l} \in E(H)$ or $v_{j}=v_{l}$ and $u_{i} u_{k} \in E(G)$. For the convenience of the following discussions, we will use the following notations in the proofs of Theorems 4 and 5. In the graph $G \square H$, the vertex $\left(u_{i}, v_{j}\right) \in V(G) \times V(H)$ is represented by $w_{i, j}$. For $j=1,2, \ldots, n$, we use $G_{j}$ to denote the subgraph of $G \square H$ induced by the vertices $w_{i, j}(i=1,2, \ldots, m)$.

Note 1. The graphs $G, G_{1}, G_{2}, \ldots, G_{n}$ are isomorphic, and for each $i(i=1,2, \ldots, m)$, the vertices $u_{i} \in V(G), w_{i, 1} \in V\left(G_{1}\right)$, $w_{i, 2} \in V\left(G_{2}\right), \ldots, w_{i, n} \in V\left(G_{n}\right)$ are the corresponding vertices under these isomorphisms.

Also, we use $E_{j}$ to denote $E\left(G_{j}\right)$; i.e., $E_{j}$ is the set of all edges in $G_{j}$. For $1 \leq j<l \leq n$ and $v_{j} v_{l} \in E(H)$, we use $E_{j, l}$ to denote the set $\left\{w_{i, j} w_{i, l} \mid i=1,2, \ldots, m\right\}$, i.e., $E_{j, l}$ is the set of all edges joining the vertices in $G_{j}$ and the vertices in $G_{l}$. We see that $E(G \square H)$ is the disjoint union of $E_{j}(j=1,2, \ldots, n)$ and $E_{j, l}\left(1 \leq j<l \leq n, v_{j} v_{l} \in E(H)\right)$.

The notations for the vertices $w_{i, j}$, the subgraphs $G_{j}$, and the edge sets $E_{j}, E_{j, l}$ of $G \square H$ will be used in the proofs of Theorems 4 and 5 .

Theorem 4. Let $G$ be a regular and uniformly $\mathbb{R}$-antimagic graph. Then, $G \square K_{n}(n \geq 2)$ is also regular and uniformly $\mathbb{R}$-antimagic. 
Proof. Since both $G$ and $K_{n}$ are regular, it is trivial that $G \square K_{n}$ is regular. Since $G$ is uniformly $\mathbb{R}$-antimagic, we assume that $u_{1}, u_{2}, \ldots, u_{m}(m \geq 3)$ is the sequence of vertices of $G$ with the uniformly $\mathbb{R}$-antimagic property. We see that the edge set $E\left(G \square K_{n}\right)$ is the union of $E_{j}(j=1,2, \ldots, n)$ and $E_{j, l}(1 \leq j<l \leq n)$.

Now, we prove that $G \square K_{n}(n \geq 2)$ is uniformly $\mathbb{R}$-antimagic. Let $A \subseteq \mathbb{R}$ with $|A|=\left|E\left(G \square K_{n}\right)\right|$ be arbitrarily given. Define $g$ to be an edge labeling of $G \square K_{n}$ with labels in $A$ by the following three rules:

Rule 1. For $j=1,2, \ldots, n-1, \quad E_{j} \prec E_{j, j+1} \prec E_{j, j}$ $+2 \prec \cdots \prec E_{j, n} \prec E_{j+1}$.

Rule 2. For $1 \leq j<l \leq n$, and for $i=1,2, \ldots, m-1$, $g\left(w_{i, j} w_{i, l}\right)<g\left(w_{i+1, j} w_{i+1, l}\right) \quad$ (i.e., $\quad g\left(w_{1, j} w_{1, l}\right)<g$ $\left.\left(w_{2, j} w_{2, l}\right)<g\left(w_{3, j} w_{3, l}\right)<\cdots<g\left(w_{m, j} w_{m, l}\right)\right)$.

Rule 3. For $j=1,2, \ldots, n$, and for $i=1,2, \ldots, m-1$, $g_{E_{j}}^{+}\left(w_{i, j}\right)<g_{E_{j}}^{+}\left(w_{i+1, j}\right)$ (i.e., $g_{E_{j}}^{+}\left(w_{1, j}\right)<g_{E_{j}}^{+}\left(w_{2, j}\right)<g_{E_{j}}^{+}$ $\left.\left(w_{3, j}\right)<\cdots<g_{E_{j}}^{+}\left(w_{m, j}\right)\right)$.

The edge labeling $g$ with labels in $A$ can have Rule 3 derived from the fact that the sequence of vertices $u_{1}, u_{2}, \ldots, u_{m}$ has the uniformly $\mathbb{R}$-antimagic property in $G$ and the fact stated in Note 1.
Claim 1. For $j=1,2, \ldots, n, g^{+}\left(w_{1, j}\right)<g^{+}\left(w_{2, j}\right)<g^{+}\left(w_{3, j}\right)$ $<\cdots<g^{+}\left(w_{m, j}\right)$.

Check of Claim 1 . We need to show $g^{+}\left(w_{i, j}\right)<g^{+}\left(w_{i+1, j}\right)$ for $i=1,2, \ldots, m-1$.

Let $J=\{1,2, \ldots, n\}$. Note that

$$
\begin{gathered}
g^{+}\left(w_{i, j}\right)=g_{E_{j}}^{+}\left(w_{i, j}\right)+\sum_{l \in J-\{j\}} g\left(w_{i, j} w_{i, l}\right), \\
g^{+}\left(w_{i+1, j}\right)=g_{E_{j}}^{+}\left(w_{i+1, j}\right)+\sum_{l \in J-\{j\}} g\left(w_{i+1, j} w_{i+1, l}\right) .
\end{gathered}
$$

By Rule 3, $g_{E_{j}}^{+}\left(w_{i, j}\right)<g_{E_{j}}^{+}\left(w_{i+1, j}\right)$.

By Rule 2, for $1 \leq j<l \leq n, g\left(w_{i, j} w_{i, l}\right)<g\left(w_{i+1, j} w_{i+1, l}\right)$, it implies

$$
\sum_{l \in J-\{j\}} g\left(w_{i, j} w_{i, l}\right)<\sum_{l \in J-\{j\}} g\left(w_{i+1, j} w_{i+1, l}\right) .
$$

Thus, $g^{+}\left(w_{i, j}\right)<g^{+}\left(w_{i+1, j}\right)$, which completes the Check of Claim 1.

Claim 2. For $j=1,2, \ldots, n-1, g^{+}\left(w_{m, j}\right)<g^{+}\left(w_{1, j+1}\right)$. Check of Claim 2. Let $J=\{1,2, \ldots, n\}$. Note that

$$
\begin{aligned}
g^{+}\left(w_{m, j}\right) & =g_{E_{j}}^{+}\left(w_{m, j}\right)+\sum_{k \in J-\{j\}} g\left(w_{m, j} w_{m, k}\right) \\
& =g_{E_{j}}^{+}\left(w_{m, j}\right)+g\left(w_{m, j} w_{m, j+1}\right)+\sum_{k \in J-\{j, j+1\}} g\left(w_{m, k} w_{m, j}\right), \\
g^{+}\left(w_{1, j+1}\right) & =g_{E_{j+1}^{+}}\left(w_{1, j+1}\right)+\sum_{k \in J-\{j+1\}} g\left(w_{1, j+1} w_{1, k}\right) \\
& =g_{E_{j+1}^{+}}\left(w_{1, j+1}\right)+g\left(w_{1, j} w_{1, j+1}\right)+\sum_{k \in J-\{j, j+1\}} g\left(w_{1, k} w_{1, j+1}\right) .
\end{aligned}
$$

Let $A_{1}=E_{G_{j}}\left(w_{m, j}\right) \subseteq E_{j}, \quad A_{2}=\left\{w_{m, j} w_{m, j+1}\right\} \subseteq E_{j, j+1}$, $B_{1}=E_{G_{j+1}}\left(w_{1, j+1}\right) \subseteq E_{j+1}, B_{2}=\left\{w_{1, j} w_{1, j+1}\right\} \subseteq E_{j, j+1}$. Thus,

$$
\begin{gathered}
\sum_{e \in A_{1} \cup A_{2}} g(e)=g_{E_{j}}^{+}\left(w_{m, j}\right)+g\left(w_{m, j} w_{m, j+1}\right), \\
\sum_{e \in B_{1} \cup B_{2}} g(e)=g_{E_{j+1}^{+}}\left(w_{1, j+1}\right)+g\left(w_{1, j} w_{1, j+1}\right) .
\end{gathered}
$$

By Rule 1, $E_{j} \prec E_{j, j+1} \prec E_{j+1}$. Since $A_{1} \subseteq E_{j}, B_{1} \subseteq E_{j+1}$, $A_{2}, B_{2} \subseteq E_{j, j+1}$, we have $A_{1} \prec B_{1} \cup B_{2}$ and $A_{2} \prec B_{1}$. Also, note $\left|A_{1}\right|=\left|B_{1}\right|, \quad\left|A_{2}\right|=\left|B_{2}\right|=1$. Thus, by Lemma 1, $\sum_{e \in A_{1} \cup A_{2}} g(e)<\sum_{e \in B_{1} \cup B_{2}} g(e)$. Hence,

$$
g_{E_{j}}^{+}\left(w_{m, j}\right)+g\left(w_{m, j} w_{m, j+1}\right)<g_{E_{j+1}^{+}}\left(w_{1, j+1}\right)+g\left(w_{1, j} w_{1, j+1}\right) \text {. }
$$

By Rule 1, $E_{k, j} \prec E_{k, j+1}$ if $k<j$, and $E_{j, k} \prec E_{j+1, k}$ if $k>j+1$, and we see that $w_{m, k} w_{m, j} \in E_{k, j}, w_{1, k} w_{1, j+1} \in E_{k, j+1}$. Thus, $g\left(w_{m, k} w_{m, j}\right)<g\left(w_{1, k} w_{1, j+1}\right)$, which implies

$$
\sum_{k \in J-\{j, j+1\}} g\left(w_{m, k} w_{m, j}\right)<\sum_{k \in J-\{j, j+1\}} g\left(w_{1, k} w_{1, j+1}\right) .
$$

Combining (20) and (21), we obtain $g^{+}\left(w_{m, j}\right)<g^{+}$ $\left(w_{1, j+1}\right)$. This completes the Check of Claim 2 .

From Claims 1 and 2, we obtain

$$
\begin{aligned}
g^{+}\left(w_{1,1}\right) & <g^{+}\left(w_{2,1}\right)<\cdots<g^{+}\left(w_{m, 1}\right) \\
& <g^{+}\left(w_{1,2}\right)<g^{+}\left(w_{2,2}\right)<\cdots<g^{+}\left(w_{m, 2}\right) \\
& <g^{+}\left(w_{1,3}\right)<g^{+}\left(w_{2,3}\right)<\cdots<g^{+}\left(w_{m, 3}\right) \\
& <\cdots<\cdots<\cdots \\
& <g^{+}\left(w_{1, n}\right)<g^{+}\left(w_{2, n}\right)<\cdots<g^{+}\left(w_{m, n}\right) .
\end{aligned}
$$

We also see that the order of the vertices $w_{1,1}, w_{2,1}, w_{3,1}$, $\ldots, w_{m, 1}, w_{1,2}, w_{2,2}, w_{3,2}, \ldots, w_{m, 2}, w_{1,3}, w_{2,3}, w_{3,3}, \ldots, w_{m, 3}$, $w_{1,4}, \ldots, w_{m, n-1}, w_{1, n}, w_{2, n}, w_{3, n}, \ldots, w_{m, n}$ satisfying the aforementioned strict inequalities is independent of the chosen $A \subseteq \mathbb{R}$ with $|A|=\left|G \square K_{n}\right|$. Thus, $G \square K_{n}(n \geq 2)$ is uniformly $\mathbb{R}$-antimagic. 
It has been proved that Cartesian product of two or more cycles is antimagic [7]. We further propose that $G \square C_{n}$ is (uniformly) $\mathbb{R}$-antimagic where $G$ is a regular and uniformly $\mathbb{R}$-antimagic graph. In $G \square C_{n}$, the labels we use are in each subset $A$ of real numbers with $|A|=|E(G)|$ and the labels used in $[7,8]$ are in $\{1,2, \ldots,|E(G)|\}$. Because of the difference in labels, we have to modify the order of labelings that are different from those in $[7,8]$. We use some strategies in the construction of labelings.

Theorem 5. Let $G$ be a regular and uniformly $\mathbb{R}$-antimagic graph. Then, $G \square C_{n}$ is also regular and uniformly $\mathbb{R}$-antimagic.

Proof. Since both $G$ and $C_{n}$ are regular, it is trivial that $G \square C_{n}$ is regular. Now, we show that $G \square C_{n}$ is uniformly $\mathbb{R}$-antimagic. By Theorem $4, G \square K_{3}$ is uniformly $\mathbb{R}$-antimagic. Thus, $G \square C_{3}$ is uniformly $\mathbb{R}$-antimagic. Using Theorem 4 twice, we see that $\left(G \square K_{2}\right) \square K_{2}$ is uniformly $\mathbb{R}$-antimagic. Thus, $G \square C_{4}$ is uniformly $\mathbb{R}$-antimagic since $\left(G \square K_{2}\right) \square K_{2}$ is isomorphic to $G \square C_{4}$. We assume that $n \geq 5$.

Assume that the cycle $C_{n}$ has vertex set $V\left(C_{n}\right)=$ $\left\{v_{1}, v_{2}, \ldots, v_{n}\right\}$ and the edge set $E\left(C_{n}\right)=\left\{v_{1} v_{2}\right\} \cup\left\{v_{i}\right.$ $\left.v_{i+2} \mid i=1,2, \ldots, n-2\right\} \cup\left\{v_{n-1} v_{n}\right\}$. We use the notations for the vertices, subgraphs, and edge sets of $G \square H$ which are defined in Theorem 4 , where $H$ is now taken to be $C_{n}$. We see that the edge set $E\left(G \square C_{n}\right)$ is the union of $E_{j}(j=1,2, \ldots, n)$ and $E_{1,2}, E_{j, j+2}(j=1,2, \ldots, n-2), E_{n-1, n}$.

Now, we prove that $G \square C_{n}$ is uniformly $\mathbb{R}$-antimagic. Since $G$ is uniformly $\mathbb{R}$-antimagic, we assume that $u_{1}, u_{2}, \ldots, u_{m}(m \geq 3)$ is the sequence of vertices of $G$ with the uniformly $\mathbb{R}$-antimagic property. Let $A \subseteq \mathbb{R}$ with $|A|=$ $\left|E\left(G \square C_{n}\right)\right|$ be arbitrarily given. Define $g$ to be an edge labeling of $G \square C_{n}$ with labels in $A$ by the following three rules:

Rule 4. Rules of $\prec$ on $G \square C_{n}$.

(a) $E_{1} \prec E_{1,2} \prec E_{2}$,

(b) for $j=2,3, \ldots, n-2, E_{j} \prec E_{j-1, j+1} \prec E_{j+1}$,

(c) $E_{n-1} \prec E_{n-2, n} \prec E_{n-1, n} \prec E_{n} \quad$ (hence $E_{1} \prec E_{1,2}$ $\prec E_{2} \prec E_{1,3} \quad \prec E_{3} \prec E_{2,4} \prec E_{4} \prec E_{3,5} \prec E_{5}$ $\prec \cdots \prec E_{n-3} \prec E_{n-4, n-2} \prec \quad E_{n-2}$ $\left.\prec E_{n-3, n-1} \prec E_{n-1} \prec E_{n-2, n} \prec E_{n-1, n} \prec E_{n}\right)$.

Rule 5. For $v_{j} v_{l} \in E\left(C_{n}\right), g\left(w_{1, j} w_{1, l}\right) \quad<g\left(w_{2, j}\right.$ $\left.w_{2, l}\right)<g\left(w_{3, j} w_{3, l}\right)<\cdots<g\left(w_{m, j} w_{m, l}\right)$.

Rule 6. For $j=1,2, \ldots, n$, we have $g_{E_{j}}^{+}\left(w_{1, j}\right)$ $<g_{E_{j}}^{+}\left(w_{2, j}\right)<g_{E_{j}}^{+}\left(w_{3, j}\right)<\cdots<g_{E_{j}}^{+}\left(w_{m, j}\right)$.

The edge labeling $g$ with labels in $A$ can have Rule 6 derived from the fact that the sequence of vertices $u_{1}, u_{2}, \ldots, u_{m}$ has the uniformly $\mathbb{R}$-antimagic property in $G$ and the fact stated in Note 1.

Claim 3. For $j=1,2, \ldots, n, g^{+}\left(w_{1, j}\right)<g^{+}\left(w_{2, j}\right)<g^{+}\left(w_{3, j}\right)$ $<\cdots<g^{+}\left(w_{m, j}\right)$.

Check of Claim 3.

We need to show $g^{+}\left(w_{i, j}\right)<g^{+}\left(w_{i+1, j}\right)$ for $i=1,2$, $\ldots, m-1$. Note that

$$
\begin{array}{r}
g^{+}\left(w_{i, j}\right)=g_{E_{j}}^{+}\left(w_{i, j}\right)+\sum_{v_{j} v_{l} \in E\left(C_{n}\right)} g\left(w_{i, j} w_{i, l}\right), \\
g^{+}\left(w_{i+1, j}\right)=g_{E_{j}}^{+}\left(w_{i+1, j}\right)+\sum_{v_{j} v_{l} \in E\left(C_{n}\right)} g\left(w_{i+1, j} w_{i+1, l}\right) .
\end{array}
$$

By Rule 6, $g_{E_{j}}^{+}\left(w_{i, j}\right)<g_{E_{j}}^{+}\left(w_{i+1, j}\right)$.

From Rule 5, we obtain that for fixed $i$, $i=1,2, \ldots, m-1$,

$$
\sum_{v_{j} v_{l} \in E\left(C_{n}\right)} g\left(w_{i, j} w_{i, l}\right)<\sum_{v_{j} v_{l} \in E\left(C_{n}\right)} g\left(w_{i+1, j} w_{i+1, l}\right) .
$$

Claim 3.

Thus, $g^{+}\left(w_{i, j}\right)<g^{+}\left(w_{i+1, j}\right)$. This completes the Check of

Claim 4. For $j=1,2, \ldots, n-1, g^{+}\left(w_{m, j}\right)<g^{+}\left(w_{1, j+1}\right)$.

Check of Claim 4. We distinguish five cases: Case 3, $j=1$; Case $4, j=2$; Case $5, j=3,4, \ldots, n-3$; Case 6 , $j=n-2 ;$ and Case $7, j=n-1$.

Case 3. $j=1$.

We need to show that $g^{+}\left(w_{m, 1}\right)<g^{+}\left(w_{1,2}\right)$. Let $A_{1}=$ $E_{G_{1}}\left(w_{m, 1}\right)$ and $A_{2}=\left\{w_{m, 1} w_{m, 2}\right\}$. Then,

$$
g^{+}\left(w_{m, 1}\right)=g\left(w_{m, 1} w_{m, 3}\right)+\sum_{e \in A_{1} \cup A_{2}} g(e) .
$$

Let $B_{1}=E_{G_{2}}\left(w_{1,2}\right)$ and $B_{2}=\left\{w_{1,1} w_{1,2}\right\}$. Then,

$$
g^{+}\left(w_{1,2}\right)=g\left(w_{1,2} w_{1,4}\right)+\sum_{e \in B_{1} \cup B_{2}} g(e)
$$

From Rule 4, $E_{1} \prec E_{1,2} \prec E_{2} \prec E_{1,3} \prec E_{2,4}$. Since $E_{1,3} \prec E_{2,4}$, we have

$$
g\left(w_{m, 1} w_{m, 3}\right)<g\left(w_{1,2} w_{1,4}\right) .
$$

Since $E_{1} \prec E_{1,2} \prec E_{2}, A_{1} \subseteq E_{1}, A_{2}, B_{2} \subseteq E_{1,2}, B_{1} \subseteq E_{2}$, we have $A_{1} \prec B_{1} \cup B_{2}, A_{2} \prec B_{1}$. Since $G$ is regular, we have $\left|A_{1}\right|=\left|B_{1}\right|$. Trivially, $\left|A_{2}\right|=\left|B_{2}\right|=1$. Thus, by Lemma 1 ,

$$
\sum_{e \in A_{1} \cup A_{2}} g(e)<\sum_{e \in B_{1} \cup B_{2}} g(e) .
$$

From the aforementioned, we obtain $g^{+}\left(w_{m, 1}\right)$ $<g^{+}\left(w_{1,2}\right)$.

Case 4. $j=2$.

We need to show that $g^{+}\left(w_{m, 2}\right)<g^{+}\left(w_{1,3}\right)$. Note that

$$
\begin{aligned}
& g^{+}\left(w_{m, 2}\right)=g_{E_{2}}^{+}\left(w_{m, 2}\right)+g\left(w_{m, 1} w_{m, 2}\right)+g\left(w_{m, 2} w_{m, 4}\right), \\
& g^{+}\left(w_{1,3}\right)=g_{E_{3}}^{+}\left(w_{1,3}\right)+g\left(w_{1,1} w_{1,3}\right)+g\left(w_{1,3} w_{1,5}\right) .
\end{aligned}
$$

Since $E_{2} \prec E_{3}$ and $G_{2}$ and $G_{3}$ are regular with the same degree, we have

$$
g_{E_{2}}^{+}\left(w_{m, 2}\right)<g_{E_{3}}^{+}\left(w_{1,3}\right)
$$

Since $E_{1,2} \prec E_{1,3}$, we have 


$$
g\left(w_{m, 1} w_{m, 2}\right)<g\left(w_{1,1} w_{1,3}\right) .
$$

Since $E_{2,4} \prec E_{3,5}$, we have

$$
g\left(w_{m, 2} w_{m, 4}\right)<g\left(w_{1,3} w_{1,5}\right)
$$

Thus, we obtain $g^{+}\left(w_{m, 2}\right)<g^{+}\left(w_{1,3}\right)$.

Case 5. $j=3,4, \ldots, n-3$.

We need to show that $g^{+}\left(w_{m, j}\right)<g^{+}\left(w_{1, j+1}\right)$. For $n=5$, we do not need to consider this case. Assume that $n \geq 6$. Note that

$$
\begin{aligned}
g^{+}\left(w_{m, j}\right) & =g_{E_{j}}^{+}\left(w_{m, j}\right)+g\left(w_{m, j-2} w_{m, j}\right)+g\left(w_{m, j} w_{m, j+2}\right), \\
g^{+}\left(w_{1, j+1}\right) & =g_{E_{j+1}}^{+}\left(w_{1, j+1}\right)+g\left(w_{1, j-1} w_{1, j+1}\right)+g\left(w_{1, j+1} w_{1, j+3}\right) .
\end{aligned}
$$

From Rule 4(b), we have $E_{j} \prec E_{j-1, j+1} \prec E_{j+1} \prec E_{j, j+2}$ for $2 \leq j \leq n-3$. Since $E_{j} \prec E_{j+1}$ and $G_{j}$ and $G_{j+1}$ are regular with the same degree, we have

$$
g_{E_{j}}^{+}\left(w_{m, j}\right)<g_{E_{j+1}}^{+}\left(w_{1, j+1}\right) \text {. }
$$

Since $E_{j-2, j} \prec E_{j-1, j+1}$, we have

$$
g\left(w_{m, j-2} w_{m, j}\right)<g\left(w_{1, j-1} w_{1, j+1}\right) .
$$

Since $E_{j, j+2} \prec E_{j+1, j+3}$, we have

$$
g\left(w_{m, j} w_{m, j+2}\right)<g\left(w_{1, j+1} w_{1, j+3}\right) .
$$

Accordingly, we obtain $g^{+}\left(w_{m, j}\right)<g^{+}\left(w_{1, j+1}\right)$.

Case 6. $j=n-2$.

We need to show that $g^{+}\left(w_{m, n-2}\right)<g^{+}\left(w_{1, n-1}\right)$. Note that

$$
\begin{aligned}
g^{+}\left(w_{m, n-2}\right)= & g_{E_{n-2}}^{+}\left(w_{m, n-2}\right)+g\left(w_{m, n-4} w_{m, n-2}\right) \\
& +g\left(w_{m, n-2} w_{m, n}\right), \\
g^{+}\left(w_{1, n-1}\right)= & g_{E_{n-1}}^{+}\left(w_{1, n-1}\right)+g\left(w_{1, n-3} w_{1, n-1}\right) \\
& +g\left(w_{1, n-1} w_{1, n}\right) .
\end{aligned}
$$

Also note that $E_{n-4, n-2} \prec E_{n-2} \prec E_{n-3, n-1} \prec E_{n-1}$. Since $w_{m, n-4} w_{m, n-2} \in E_{n-4, n-2}, w_{1, n-3} w_{1, n-1} \in E_{n-3, n-1}$, we have

$$
g\left(w_{m, n-4} w_{m, n-2}\right)<g\left(w_{1, n-3} w_{1, n-1}\right) \text {. }
$$

Since $E_{G_{n-2}}\left(w_{m, n-2}\right) \subseteq E_{n-2}, E_{G_{n-1}}\left(w_{1, n-1}\right) \subseteq E_{n-1}$, we have

$$
g_{E_{n-2}}^{+}\left(w_{m, n-2}\right)<g_{E_{n-1}}^{+}\left(w_{1, n-1}\right) \text {. }
$$

Furthermore, $E_{n-2, n} \prec E_{n-1, n}$, this implies

$$
g\left(w_{m, n-2} w_{m, n}\right)<g\left(w_{1, n-1} w_{1, n}\right) .
$$

Hence, we obtain $g^{+}\left(w_{m, n-2}\right)<g^{+}\left(w_{1, n-1}\right)$.

Case 7. $j=n-1$.

We need to show that $g^{+}\left(w_{m, n-1}\right)<g^{+}\left(w_{1, n}\right)$. Let $A_{1}=$ $E_{G_{n-1}}\left(w_{m, n-1}\right)$ and $A_{2}=\left\{w_{m, n-1} w_{m, n}\right\}$. Then,

$$
g^{+}\left(w_{m, n-1}\right)=g\left(w_{m, n-3} w_{m, n-1}\right)+\sum_{e \in A_{1} \cup A_{2}} g(e) .
$$

Let $B_{1}=E_{G_{n}}\left(w_{1, n}\right)$ and $B_{2}=\left\{w_{1, n-1} w_{1, n}\right\}$. Then,

$$
g^{+}\left(w_{1, n}\right)=g\left(w_{1, n-2} w_{1, n}\right)+\sum_{e \in B_{1} \cup B_{2}} g(e) .
$$

Note that $E_{n-3, n-1} \prec E_{n-1} \prec E_{n-2, n} \prec E_{n-1, n} \prec E_{n}$. From $E_{n-3, n-1} \prec E_{n-2, n} \quad$ and $w_{m, n-3} w_{m, n-1} \in E_{n-3, n-1}$, $w_{1, n-2} w_{1, n} \in E_{n-2, n}$, we have

$$
g\left(w_{m, n-3} w_{m, n-1}\right)<g\left(w_{1, n-2} w_{1, n}\right) .
$$

From $E_{n-1} \prec E_{n-1, n} \prec E_{n}$ and $A_{1} \subseteq E_{n-1}, \quad A_{2} \subseteq E_{n-1, n}$, $B_{1} \subseteq E_{n}, B_{2} \subseteq E_{n-1, n}$, we have $A_{1} \prec B_{1} \cup B_{2}$ and $A_{2} \prec B_{1}$. Since $G$ is regular, we have $\left|A_{1}\right|=\left|B_{1}\right|$. Trivially, $\left|A_{2}\right|=\left|B_{2}\right|=1$. Thus, by Lemma 1,

$$
\sum_{e \in A_{1} \cup A_{2}} g(e)<\sum_{e \in B_{1} \cup B_{2}} g(e) .
$$

Therefore, we obtain $g^{+}\left(w_{m, n-1}\right)<g^{+}\left(w_{1, n}\right)$.

These complete the Check of Claim 4.

From Claims 3 and 4, we obtain

$$
\begin{aligned}
g^{+}\left(w_{1,1}\right) & <g^{+}\left(w_{2,1}\right)<\cdots<g^{+}\left(w_{m, 1}\right) \\
& <g^{+}\left(w_{1,2}\right)<g^{+}\left(w_{2,2}\right)<\cdots<g^{+}\left(w_{m, 2}\right) \\
& <g^{+}\left(w_{1,3}\right)<g^{+}\left(w_{2,3}\right)<\cdots<g^{+}\left(w_{m, 3}\right) \\
& <\cdots<\cdots<\cdots \\
& <g^{+}\left(w_{1, n}\right)<g^{+}\left(w_{2, n}\right)<\cdots<g^{+}\left(w_{m, n}\right) .
\end{aligned}
$$

We also see that the order of the vertices $w_{1,1}, w_{2,1}, w_{3,1}$, $\ldots, w_{m, 1}, w_{1,2}, w_{2,2}, w_{3,2}, \ldots, w_{m, 2}, w_{1,3}, w_{2,3}, w_{3,3}, \ldots, w_{m, 3}$, $w_{1,4}, \ldots, w_{m, n-1}, w_{1, n}, w_{2, n}, w_{3, n}, \ldots, w_{m, n}$ satisfying the aforementioned strict inequalities is independent of the chosen $A \subseteq \mathbb{R}$ with $|A|=\left|E\left(G \square C_{n}\right)\right|$. Thus, $G \square C_{n}$ is uniformly $\mathbb{R}$-antimagic. This completes the proof of the theorem.

The following corollaries derive directly from Theorems 4 and 5.

Corollary 1. The graph $G_{1} \square G_{2} \square \cdots \square G_{n}$ ( $n \geq 2$ ) is uniformly $\mathbb{R}$-antimagic, where $G_{1}$ is regular and uniformly $\mathbb{R}$-antimagic, and for $i \geq 2$, each $G_{i}$ is a complete graph of order $\geq 2$ or a cycle.

Corollary 2. The graph $G_{1} \square G_{2} \square \cdots \square G_{n}$ ( $\left.n \geq 2\right)$ is uniformly $\mathbb{R}$-antimagic, where each $G_{i}$ is a complete graph of order $\geq 2$ or a cycle.

Proof. Each $G_{i}$ is a complete graph of order $\geq 2$ or a cycle.

Case 8. Some $G_{i} \neq K_{2}$.

Without loss of generality, assume $G_{1} \neq K_{2}$. Then, $G_{1}$ is a cycle or a complete graph of order $\geq 3$. By Theorems 2 and $3, G_{1}$ is uniformly $\mathbb{R}$-antimagic. Then, the corollary derives from Corollary 1. 
Case 9. $G_{i}=K_{2}$ for $i=1,2, \ldots, n$.

Since $K_{2} \square K_{2} \cong C_{4}$, by Theorem $2, G_{1} \square G_{2}$ is uniformly $\mathbb{R}$-antimagic. Again, the corollary derives from Corollary 1.

Note that the hypercube $Q_{n}$ is isomorphic to $G_{1} \square G_{2} \square \cdots \square G_{n}$, where each $G_{i}=K_{2}$ for $i=1,2, \ldots, n$. The following corollary derives from Corollary 2 .

Corollary 3. Hypercube $Q_{n}(n \geq 2)$ is uniformly $\mathbb{R}$-antimagic.

\section{Conclusions}

In this paper, we propose the notion of $\mathbb{R}$-antimagic graph. This is a generalization of $\mathbb{R}^{+}$-antimagic graph. Every $\mathbb{R}$-antimagic graph is $\mathbb{R}^{+}$-antimagic, and every $\mathbb{R}^{+}$-antimagic is antimagic. Not all $\mathbb{R}^{+}$-antimagic graphs (e.g., stars and $\left.P_{n}, n=3,4,5\right)$ are $\mathbb{R}$-antimagic.

In Section 2, we show that wheels, cycles, and complete graphs of order $\geq 3$ are $\mathbb{R}$-antimagic. Let $G$ be a complete graph (except $K_{1}$ ) or a cycle with $V(G)=\left\{u_{1}, u_{2}, \ldots, u_{n}\right\}$. We have found that all the vertices of $G$ can be listed as $u_{1}, u_{2}, \ldots, u_{n}$ such that for every $A \subseteq \mathbb{R}$ with $|A|=|E(G)|$, there is an edge labeling $f$ of $G$ with labels in $A$ such that $f^{+}\left(u_{1}\right)<f^{+}\left(u_{2}\right)<\cdots<f^{+}\left(u_{n}\right)$. The property we call uniformly $\mathbb{R}$-antimagic property is independent of the choice of the subset $A$ of $\mathbb{R}$. We have found some graphs with uniformly $\mathbb{R}$-antimagic property.

We use labelings modified from those in $[7,8]$ and make them more systematic in this paper. The proofs in this paper provide efficient algorithms for finding edge labelings of Cartesian products of cycles and complete graphs. Our contribution is to quickly find the edge labelings of Cartesian products of cycles and complete graphs through the algorithms we constructed. It has been proved the Cartesian products $G_{1} \square G_{2} \square \cdots \square G_{n}(n \geq 2)$ of $G_{1}, G_{2}, \ldots, G_{n}$ are (uniformly) $\mathbb{R}$-antimagic if each $G_{i}$ is either a complete graph (except $K_{1}$ ) or a cycle in Section 3.

We construct some classes of uniformly $\mathbb{R}$-antimagic graphs through Cartesian products. Some join graphs which are antimagic have been proved in $[13,14]$. In [13], they use the way of listing edges in [9] to show that a class of join graphs are antimagic. It makes the method of labelings in this paper more plausible.

We end this paper with the following observation: every $\mathbb{R}^{+}$-antimagic graph is also $\mathbb{R}$-antimagic if the graph is regular. In further studies, we will propose $\mathbb{R}$-antimagicness of more regular graphs (e.g., Petersen graph). Also, we will generalize the research results in this paper in the proposals of Cartesian product of some other regular graphs.

\section{Data Availability}

No data were used to support this study.

\section{Conflicts of Interest}

The authors declare no conflicts of interest.

The authors declare no conflicts of interest.

\section{References}

[1] N. Hartsfield and G. Ringel, Pearls in Graph Theory, Academic Press, Inc., Boston, MA, USA, 1990.

[2] D. W. Cranston, "Regular bipartite graphs are antimagic," Journal of Graph Theory, vol. 60, no. 3, pp. 173-182, 2009.

[3] D. W. Cranston, Y.-C. Liang, and X. Zhu, "Regular graphs of odd degree are antimagic," Journal of Graph Theory, vol. 80, no. 1, pp. 28-33, 2015.

[4] Y.-C. Liang and X. Zhu, "Antimagic labeling of cubic graphs," Journal of Graph Theory, vol. 75, no. 1, pp. 31-36, 2014.

[5] F. Chang, Y.-C. Liang, Z. Pan, and X. Zhu, "Antimagic labeling of regular graphs," Journal of Graph Theory, vol. 82, no. 4, pp. 339-349, 2016.

[6] T.-M. Wang and C.-C. Hsiao, "On anti-magic labeling for graph products," Discrete Mathematics, vol. 308, no. 16, pp. 3624-3633, 2008.

[7] T.-M. Wang, "Toroidal grids are anti-magic," Lecture Notes in Computer Science, Springer, vol. 3595, pp. 671-679, Berlin, Germany, 2005.

[8] Y. Cheng, "Lattice grids and prisms are antimagic," Theoretical Computer Science, vol. 374, no. 1-3, pp. 66-73, 2007.

[9] Y. Cheng, "A new class of antimagic Cartesian product graphs," Discrete Mathematics, vol. 308, no. 24, pp. 6441-6448, 2008.

[10] Y. Zhang and X. Sun, "The antimagicness of the Cartesian product of graphs," Theoretical Computer Science, vol. 410, no. 8-10, pp. 727-735, 2009.

[11] M. Matamala and J. Zamora, "Graphs admitting antimagic labeling for arbitrary sets of positive numbers," Discrete Applied Mathematics, vol. 281, pp. 246-251, 2020.

[12] J. L. Shang, C. Lin, and S. C. Liaw, "On the antimagic labeling of star forests," Utilitas Mathematica, vol. 97, pp. 373-385, 2015.

[13] T. Wang, M. J. Liu, and D. M. Li, "A class of antimagic join graphs," Acta Mathematica Sinica, English Series, vol. 29, no. 5, pp. 1019-1026, 2013.

[14] M. Bača, O. Phanalasy, J. Ryan, and A. SemaničováFeňovčíková, "Antimagic labelings of join graphs," Mathematics in Computer Science, vol. 9, no. 2, pp. 139-143, 2015. 